Saturation of the ion-hammering effect for large non-hydrostatic capillarity stresses in colloidal silica nanoparticles

This article has been downloaded from IOPscience. Please scroll down to see the full text article.

2011 Nanotechnology 22475302

(http://iopscience.iop.org/0957-4484/22/47/475302)

View the table of contents for this issue, or go to the journal homepage for more

Download details:

IP Address: 150.203.178.98

The article was downloaded on 02/11/2011 at 21:31

Please note that terms and conditions apply. 


\title{
Saturation of the ion-hammering effect for large non-hydrostatic capillarity stresses in colloidal silica nanoparticles
}

\author{
Giancarlo Rizza $^{1}$, Yaasiin Ramjauny ${ }^{1}$, Marc Hayoun ${ }^{1}$, \\ Sandrine Perruchas ${ }^{2}$, Thierry Gacoin ${ }^{2}$, Patrick Kluth ${ }^{3}$ and \\ Mark C Ridgway ${ }^{3}$ \\ ${ }^{1}$ Ecole Polytechnique, Laboratoire des Solides Irradiés (LSI), CEA/DSM/IRAMIS-CNRS, \\ 91128 Palaiseau Cedex, France \\ ${ }^{2}$ Ecole Polytechnique, Laboratoire de Physique de la Matière Condensée (LPMC), CNRS, \\ 91128, Palaiseau Cedex, France \\ ${ }^{3}$ Department of Electronic Materials Engineering, Research School of Physical Sciences and \\ Engineering, Australian National University, Canberra, Australia \\ E-mail: giancarlo.rizza@polytechnique.edu
}

Received 5 July 2011, in final form 9 October 2011

Published 2 November 2011

Online at stacks.iop.org/Nano/22/475302

\begin{abstract}
We investigate the role of capillarity stresses on the ion-hammering phenomenon when sub-micrometer colloidal particles are considered. To this end, nearly monodisperse, chemically synthesized silica $\left(\mathrm{SiO}_{2}\right)$ colloids $(100,300$ and $600 \mathrm{~nm})$ were irradiated at room temperature $(300 \mathrm{~K})$ with $4 \mathrm{MeV}$ Au ions for fluences up to $\Phi=1.8 \times 10^{16} \mathrm{~cm}^{-2}$. It has been taken for granted that the transverse dimension of an ion-deformable amorphous material grows exponentially with the irradiation fluence, $L(\phi)=L(0) \exp \left[A_{0} \Phi\right]$. Here, we show that for sub-micrometer particles the irradiation-induced deformation saturates for larger fluences, $L(\phi) \rightarrow$ const. The saturation fluence depends on the initial dimension of the colloidal nanoparticles: the smaller the dimension of the colloids, the lower the saturation fluence. Experimental data are successfully accounted for by having recourse to a phenomenological model first developed by Klaumünzer and further elaborated by van Dillen. We also estimate the evolution with fluence of the principal stresses inside the particles, $\sigma_{11}(\phi)=\sigma_{22}(\phi)$ and $\sigma_{33}(\phi)$, and we show that they evolve toward a steady-state value following a sigmoidal-like behavior. Furthermore, when stresses induced by the surface curvature become non-negligible the approximation often made that the deformation strain rate, $A_{0}=\mathrm{d} L / L \mathrm{~d} \Phi$, remains constant upon irradiation is no longer valid. We show that $A_{0}$ evolves with the irradiation fluence, e.g. $A_{0} \longrightarrow A(\Phi)$, and we relate this behavior to the evolution of the stresses upon irradiation. Finally, this work allows us to define the limits of the ion-hammering effect when the non-hydrostatic capillarity stresses become important.
\end{abstract}

Macroscopic plastic flow occurs in all amorphous materials when one irradiates them with heavy ions. This process is known as irradiation-induced anisotropic deformation or the ion-hammering effect. The irradiated sample shrinks in the direction of the ion beam and expands perpendicularly to it. This phenomenon was discovered in the early 1980s, almost simultaneously by Klaumünzer et al [1], and by Cartz et al [2], for two different classes of amorphous materials and in two different regions of the energy spectrum. Klaumünzer et al observed the ion-deformation of a $\mathrm{Pd}_{80} \mathrm{Si}_{20}$ metallic glass thin film when irradiated at low temperature $(80 \mathrm{~K})$ with fast heavy ions (285 MeV Kr) [1]. Successive studies have shown that: (i) the ion-hammering effect is a universal phenomenon occurring in all irradiated amorphous materials [3], (ii) the deformation increases with the electronic energy loss [4], and (iii) decreases with increasing irradiation temperature [1]. On the other hand, Cartz et al observed the deformation of ion-amorphized silicate micro-particles $(<5 \mu \mathrm{m})$ when 
irradiated at room temperature with $3 \mathrm{MeV} \mathrm{Kr}$ ions [2]. This work was recently developed by Polman's group using chemically synthesized silica colloids as a sub-microscopic model system [5]. The authors show that micrometer-sized spherical silica colloids deform into oblate spheroids with an aspect ratio that can be tuned by varying irradiation conditions and temperature. On the other hand, the authors show: (i) a roll-off effect of the colloids when irradiated at $45^{\circ}$ relative to the surface normal, and (ii) the absence of an electronic stopping power threshold value for deformation, e.g. for a planar film this is about $2 \mathrm{keV} \mathrm{nm}^{-1}$.

From a theoretical point of view, the crucial issue for the ion-hammering phenomenon is the relation of the irradiationinduced macroscopic deformation to the energy deposited within the material by the impinging ion. In particular, the ion beam induced anisotropic plastic deformation is assumed to be directly related to the track formation [6]. In this context, the irradiation-induced anisotropic deformation has been successfully described by the 'effective flow temperature approach' [7, 8]. The model states that when the ion-track temperature exceeds a certain flow temperature, $T^{*}$, complete shear stress relaxation takes place. Upon rapid cooling of the spike region, the strain increment associated with stress relaxation freezes in. The resulting strained track represents the mesoscopic defect responsible for the anisotropic deformation. Thus, the overall effect is given by the sum of the frozen-in viscous strain of individual ion impacts. Within this approach, the macroscopic viscous shear strain is calculated by having recourse to Eshelby's theory of ellipsoidal elastic inclusions in elastic isotropic media [9]. This model has been implemented by van Dillen et al by introducing the time evolution of the stresses generated during track formation as well as the temporal evolution of shear stress relaxation [10].

For an unconstrained amorphous thin film, the experimental data can be modeled by introducing the rate-of-strain tensor, $\mathrm{d} \varepsilon_{i j} / \mathrm{d} \Phi$, such that:

$$
A_{i j}^{0}=\frac{\mathrm{d} \varepsilon_{i j}}{\mathrm{~d} \Phi}
$$

where the tensor $A_{i j}^{0}=A_{0}\left(\delta_{i j}-3 a_{i} a_{j}\right)$ represents the deformation yield, or the deformation rate. $A_{0}$ represents the strain increment per unit fluence, $\delta_{i j}$ is the Kronecker delta, $a_{i}$ are the components of the unit vector $\mathbf{a}, \Phi=\varphi t$ is the ion fluence, $\varphi$ the ion flux and $t$ the irradiation time. For a planar film, the solution of equation (1) in the direction perpendicular to the beam trajectory, $L(\Phi)$, is an exponential function of the fluence, i.e. $L(\phi)=L(0) \exp \left[A_{0} \Phi\right]$.

For large colloids $(\geqslant 1 \mu \mathrm{m})$, deformation of the dimension normal to the ion beam is the same as for the unconstrained thin film. However, when the size of the colloids is reduced to values within the sub-micrometer region, iondeformation becomes strongly size dependent [11]. In this case, equation (1) fails to fit the experimental data and the complete constitutional equation must be considered [12]:

$$
\frac{\mathrm{d} \varepsilon_{i j}}{\mathrm{~d} t}=A_{i j}^{0} \frac{\mathrm{d} \Phi}{\mathrm{d} t}+\frac{1}{2 \mu} \frac{\mathrm{d}}{\mathrm{d} t}\left[\sigma_{i j}-\frac{v}{1+v} \sigma_{k k} \delta_{i j}\right]+\frac{s_{i j}}{2 \eta} .
$$

The first term describes the unconstrained anisotropic deformation process, i.e. equation (1). The second term represents the time-dependent elastic behavior of the materials, i.e. it corresponds to Hooke's law for isotropic elastic media, where $\sigma_{i j}$ are the non-hydrostatic capillarity stresses and $\mu$ and $v$ are the shear modulus and the Poisson's ratio, respectively. The third term describes the inelastic behavior of the material, i.e. the macroscopic irradiation-induced Newtonian viscous flow. Here, $s_{i j}=\left(\sigma_{i j}-\frac{1}{3} \sigma_{k k} \delta_{i j}\right)$ are the deviatoric stresses and $\eta \propto(\mathrm{d} \Phi / \mathrm{d} t)^{-1}$ is the ion-flux-dependent shear viscosity.

Equation (1) can be successfully applied for large colloids because the non-hydrostatic capillarity stresses, induced by the surface curvature, are negligible [12]. However, when the size of the colloids is reduced, these become important and the second and third terms of equation (2) must be considered. These stresses may alter the shear relaxation in the molten track and thus the deformation process. In particular, as radiationinduced Newtonian flow leads toward a hydrostatic state, for large fluences it must, in principle, counterbalance the iondeformation effect [12]. Thus, the motivation of the present work is to give an answer to the following open question: what happens when the surface curvature is so small, i.e. when the large axis of the colloids becomes so long, that the nonhydrostatic capillarity stresses turn out to be equivalent to those associated with the ion-deformation process?

\section{Experimental details}

Colloidal $\mathrm{SiO}_{2}$ nanoparticles (NPs) were synthesized following the Stöber process $[13,14]$. Tetrapropylorthosilicate (TPOS), or tetraethylorthosilicate (TEOS) in the case of the $600 \mathrm{~nm}$ NPs, were used as silica precursors which were reacted with a $\mathrm{NH}_{4} \mathrm{OH}$ solution in ethanol at room temperature. The size was controlled by changing the concentrations of the silica precursors and of $\mathrm{NH}_{4} \mathrm{OH}$. The average size of the silica colloids was estimated to be 102, 289 and $605 \mathrm{~nm}( \pm 10 \%)$, using transmission electron microscopy (TEM), field emission scanning electron microscopy (FE-SEM) and dynamic light scattering (DLS) techniques. After chemical synthesis, silica colloids were deposited, well dispersed, onto microscope grids coated with a $50 \mathrm{~nm}$ thick carbon film. Samples were then irradiated with $4 \mathrm{MeV} \mathrm{Au}$ ions at room temperature $(300 \mathrm{~K})$ and at normal incidence for fluences ranging from $1 \times 10^{14} \mathrm{~cm}^{-2}$ up to $1.8 \times 10^{16} \mathrm{~cm}^{-2}$. Experiments were performed under the same experimental conditions having recourse to the ARAMIS accelerator in Orsay (France) and the high-energy implanter in Canberra (Australia). The nuclear and the electronic stopping power of the $\mathrm{Au}$ ions in $\mathrm{SiO}_{2}$ $\left(S_{n}=1.7 \mathrm{keV} \mathrm{nm}^{-1}\right.$ and $\left.S_{\mathrm{e}}=1.9 \mathrm{keV} \mathrm{nm}^{-1}\right)$ were calculated with the SRIM 2006 code [15] and using $2.0 \mathrm{~g} \mathrm{~cm}^{-3}$ as the density of the silica. The samples were analyzed in a $300 \mathrm{keV}$ Philips CM30 microscope. TEM micrographs were processed with a slow-scan CCD camera and analyzed with the Digital micrograph program. Samples were also analyzed using a Hitachi S-4800 FE-SEM. The average size of the NPs and their size dispersion have been estimated by considering the corresponding size distribution profiles. These were obtained by analyzing at least 500 particles for each sample. Finally, to reduce the systematic error, for each microscope grid, the average size of the NPs was estimated before and after the irradiation. 

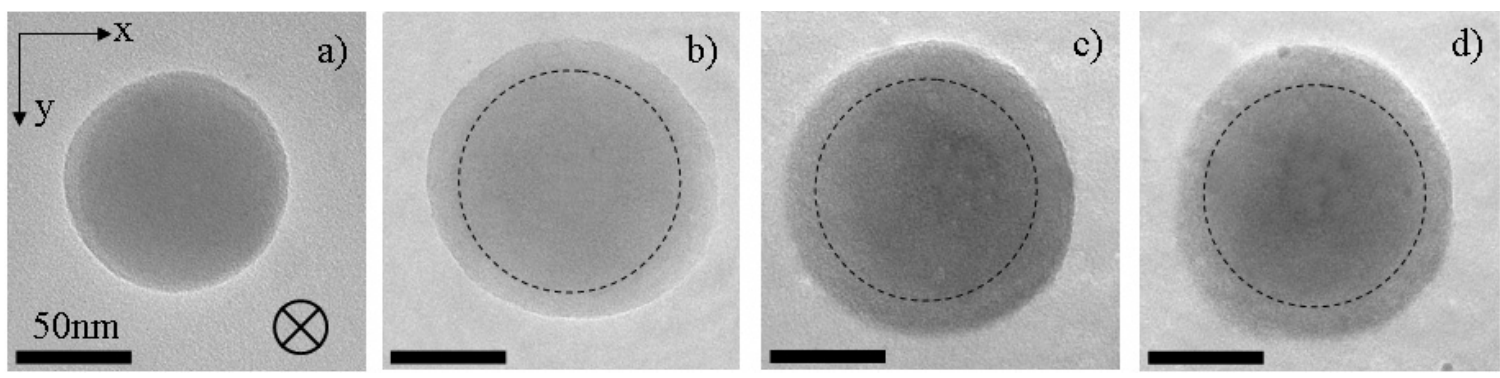

Figure 1. (a) Bright field TEM micrograph of an as-synthesized $100 \mathrm{~nm} \mathrm{SiO} 2$ colloid. (b)-(d) Bright field TEM micrographs of a $100 \mathrm{~nm}$ $\mathrm{SiO}_{2}$ colloidal NP irradiated with $4 \mathrm{MeV}$ Au ions at $300 \mathrm{~K}$ : (b) $0.5 \times 10^{16} \mathrm{~cm}^{-2}$, (c) $1.0 \times 10^{16} \mathrm{~cm}^{-2}$ and (d) $1.8 \times 10^{16} \mathrm{~cm}^{-2}$. The ion irradiation is normal to the plane, $\otimes$. The dotted circles correspond to the initial NP size. The colloids shrink along the beam ( $z$ direction) and expand normal to the beam ( $x y$ plane).
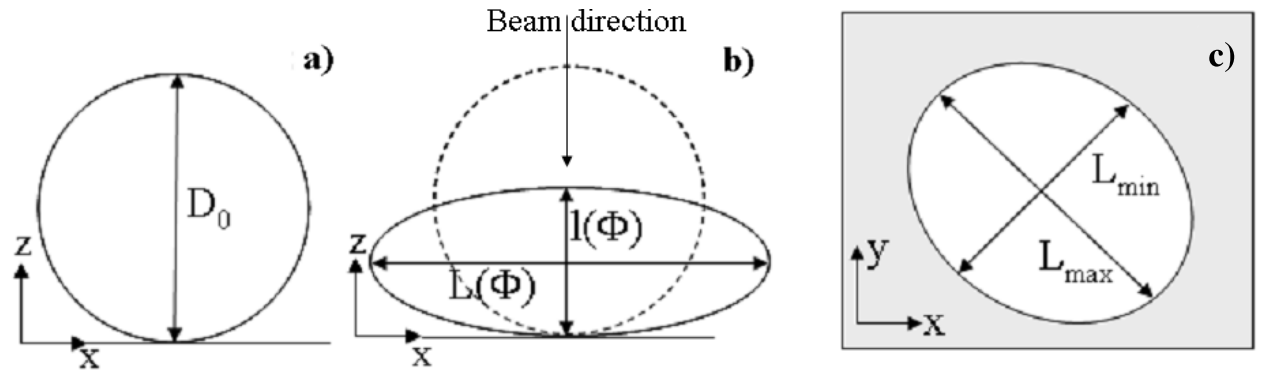

Figure 2. (a) Diameter of the as-prepared NPs, $D_{0}$. (b) Major $(L(\Phi))$ and minor $(l(\Phi))$ axes for the irradiated NPs as a function of the fluence $(\Phi)$. (c) Estimation of the major axis for imperfectly circular NPs, $L=\left(L_{\max }+L_{\min }\right) / 2$.

\section{Results}

Figure 1(a) shows an example of an as-prepared $100 \mathrm{~nm}$ silica colloid. Figures 1(b)-(d) show the anisotropic deformation of $100 \mathrm{~nm}$ nanocolloid upon irradiation with $4 \mathrm{MeV} \mathrm{Au}$ ions at room temperature for fluences up to $1.8 \times 10^{16} \mathrm{~cm}^{-2}$. The evolution with fluence for larger colloids (300 and $600 \mathrm{~nm}$ ) is similar to that of figure 1 and thus not shown here. The ion beam direction is normal to the supporting coated carbon film, i.e. perpendicular to the micrograph. In order to help the reader to appreciate the deformation process, a dotted circle, corresponding to the original dimension of the silica particle, has been drawn on the micrograph of each colloid. From the inspection of figure 1, some conclusions can be drawn: (i) for low irradiation fluences the deformation process is isotropic, i.e. the projection of the spherical particle onto the image screen (the $x y$ plane) remains a circle; whereas for larger fluences, colloids assume a slightly oblate shape. As this effect does not show a preferred orientation, it cannot be correlated to an eventual substrate deformation and must be considered as an intrinsic property of the irradiated colloids. (ii) for higher irradiation fluence, the contours of the silica colloids become less well defined and contrast fluctuations are observed.

The notation used in the paper to describe the evolution of the particles under irradiation is defined in figures 2(a)(c). $D_{0}$ is the diameter of the as-prepared NPs, $L(\Phi)$ is the dimension normal to the ion beam and $l(\Phi)$ the dimension along the ion beam as a function of the fluence, $\Phi$. For imperfectly spherical particles the value of $L(\Phi)$ is assumed to be $L(\Phi)=\left(L_{\max }(\Phi)+L_{\min }(\Phi)\right) / 2$. The evolution with

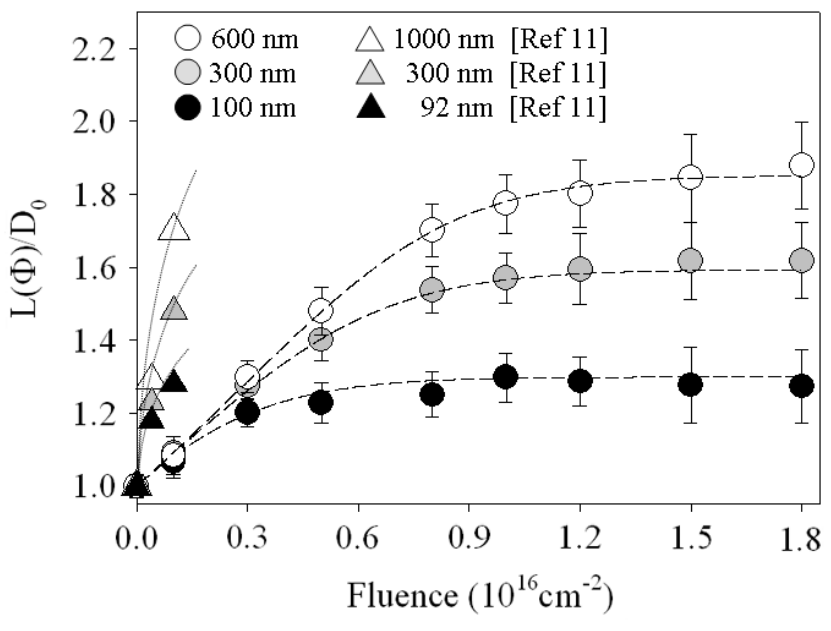

Figure 3. Evolution with fluence of the normalized transverse diameter $\left(L(\Phi) / D_{0}\right)$ as a function of the ion fluence. Full, gray and open circles represent the experimental evolution of 100, 300 and $600 \mathrm{~nm} \mathrm{SiO}{ }_{2}$ colloidal NPs, respectively. Dashed lines are the results of the numerical fitting of the parameters $A_{0}$ and $\eta_{\mathrm{RAD}}$. The fitting procedure is discussed in section 3.1. For comparison we added the evolution of $\mathrm{SiO}_{2}$ colloids as measured by van Dillen et al [11]: $1000 \mathrm{~nm} \mathrm{SiO} 2$ (open triangles), $300 \mathrm{~nm} \mathrm{SiO}_{2}$ (gray triangles) and $100 \mathrm{~nm} \mathrm{SiO}_{2}$ (full triangles).

fluence of the dimension normal to the ion beam corresponding to the different sizes of the colloids $(100,300$ and $600 \mathrm{~nm})$ is reported in figure 3. Data have been normalized to the initial particle diameter, $\left(L(\Phi) / D_{0}\right)$. Two features can be observed: 
(i) for small fluences $\left(<1 \times 10^{15} \mathrm{~cm}^{-2}\right)$ the anisotropic deformation is not size dependent; (ii) at higher ion fluences, the ion-hammering effect is observed to saturate for the three colloidal sizes. In particular, smaller colloids deform less and saturate faster than larger ones. The transverse dimension of $100 \mathrm{~nm}$ colloids (full black circles) saturates at a value of about 1.27 for fluences larger than $5 \times 10^{15} \mathrm{~cm}^{-2}$. These two values increase to about 1.6 and $1 \times 10^{16} \mathrm{~cm}^{-2}$ for $300 \mathrm{~nm}$ colloids (full gray circles), respectively. Finally, for $600 \mathrm{~nm}$ NPs (open circles), deformation evolves toward a steady-state of about 1.8 for fluences larger than $1.3 \times 10^{16} \mathrm{~cm}^{-2}$. It is worth mentioning that these fluences are more than one order of magnitude larger that those normally used for ion-hammering experiments, i.e. up to a few $10^{15} \mathrm{~cm}^{-2}$.

\section{Discussion}

\subsection{Fitting equation}

In section 2 we showed that: (i) the anisotropic deformation saturates for larger irradiation fluences and that (ii) the saturation value is size dependent. The objective of this section is to use equation (2) to model our experimental results.

For an unconstrained amorphous film (or for microparticles) the ion-deformation in the direction perpendicular to the beam, $L(\Phi)$, is an exponential function of the fluence, e.g. equation (1). However, Klaumünzer has suggested that when non-hydrostatic capillarity stresses become nonnegligible (i.e. for particles with large aspect ratios and/or for large irradiation fluences), the Newtonian flow must counterbalance the irradiation-induced deformation effect, leading to a saturation of the ion-deformation process [12]. He established a complete constitutional equation to describe the evolution of the irradiated system when stresses associated with the surface curvature, $\sigma_{i j}$, are present, i.e. equation (2). In our case, the second term of equation (2), i.e. the pure elastic contribution, can be ignored as the average elastic strain contribution perpendicular to the beam $\left(\mathrm{d} \epsilon_{11} / \mathrm{d} \Phi=\right.$ $\mathrm{d} \epsilon_{22} / \mathrm{d} \Phi \sim 10^{-20} \mathrm{~cm}^{2} /$ ion) is much smaller than the initial strain increment $A_{0} \sim 10^{-16} \mathrm{~cm}^{2} /$ ion. The value for $A_{0}$ is obtained from the fit of equation (3). It justifies, a posteriori, the previous approximation. Moreover, using the approximation that the stress components inside the colloids are uniform, the irradiation-induced deformation of the particles in the presence of the Newtonian viscous flow equation (2) can be rewritten in a more useful way as [11]:

$$
\frac{\mathrm{d} L}{\mathrm{~d} \Phi}=A_{0} L-\frac{\gamma}{3 \eta_{\mathrm{RAD}}}\left[1+\frac{L^{6}}{D_{0}^{6}}-2 \frac{D_{0}^{3}}{L^{3}}\right] .
$$

Here $A_{0}$ is the initial strain increment supposed constant, $\gamma$ is the surface energy, $L$ the major axis, $D_{0}$ the initial diameter of the colloid and $\Phi$ the fluence. The shear viscosity depends on the ion flux, i.e. $\eta \propto(\mathrm{d} \Phi / \mathrm{d} t)^{-1}$. However, to avoid this explicit dependence in equation (3) the normalized radiation-induced viscosity is considered, i.e. $\eta_{\mathrm{RAD}}=\eta \times$ $(\mathrm{d} \Phi / \mathrm{d} t)$. Thus, $\eta_{\mathrm{RAD}}$ represents the ion flux independent radiation-induced viscosity [22, 23]. The first term of equation (3) describes the deformation in the absence of stress whereas the second term is the Newtonian viscous flow resulting from the stress surface curvature. Finally, in equation (3) major $(L)$ and minor $(l)$ axes are related through volume conservation, i.e. $l=D_{0}^{3} / L^{2}$. Unfortunately, no analytical expression exists for $L(\Phi)$. Thus, we fix the parameters $A_{0}$ and $\eta_{\text {RAD }}$ and numerically compute $L(\Phi)$ from the known values $L(\Phi=0)$ and $\mathrm{d} L(\Phi=0) / \mathrm{d} \Phi$. $\Phi$ is iteratively incremented from zero by steps of $\delta \Phi=$ $1.8 \times 10^{13} \mathrm{~cm}^{-2} . L(\Phi)$ being obtained, $A(\Phi)=\mathrm{d} L / L \mathrm{~d} \Phi$ is easily calculated. The parameters $A_{0}$ and $\eta_{\mathrm{RAD}}$ have been fitted on the nine experimental values of $L(\Phi)$ for the three sizes of the silica colloids (total of 27 points) by means of the sum of the least squares of the differences between computed and experimental $L(\Phi)$. The value of this sum has been canceled using the package MERLIN that makes available several minimization algorithms in a highly interactive environment [24]. The best agreement was obtained for $A_{0}=(0.90 \pm 0.01) \times 10^{-16} \mathrm{~cm}^{2} /$ ion and $\eta_{\mathrm{RAD}}=$ $(5.4 \pm 0.3) \times 10^{22} \mathrm{~Pa}$ ion $\mathrm{cm}^{-2}$. The curves corresponding to the fitting parameters $\left(A_{0}\right.$ and $\left.\eta_{\mathrm{RAD}}\right)$ are plotted in figure 3 . In the same figure, we superpose the results of van Dillen et al [11] for $\mathrm{SiO}_{2}$ colloids in the size range of 100-1000 nm after irradiation at $85 \mathrm{~K}$ with $4 \mathrm{MeV} \mathrm{Xe}$ ions (triangles). Clearly, in the van Dillen experiment the saturation of the ion-hammering effect has not been attained yet. Thus, our observation represents the first demonstration that the irradiation-induced stress can be compensated by the macroscopic stresses induced by the curvature radius of the colloids.

Finally, if we compare our estimation for $A_{0}$ and $\eta_{\mathrm{RAD}}$ to that obtained by van Dillen, i.e. $A_{0} \sim 6.2 \times 10^{-16} \mathrm{~cm}^{2} /$ ion and $\eta_{\mathrm{RAD}}=0.9 \times 10^{22} \mathrm{~Pa}$ ions $\mathrm{cm}^{-2}$, we observe that the latter are four to five times smaller for $\eta_{\mathrm{RAD}}$ and six times larger for $A_{0}$ than ours. These observations will be discussed in the following sub-sections.

3.1.1. Radiation-induced viscosity, $\eta_{R A D}$. In section 2 we observed that our numerical fitting yields a value of $\eta_{\text {RAD }}$ which is larger than the one obtained by van Dillen. We recall that our experiments were performed at room temperature (300 K) using $4 \mathrm{MeV} \mathrm{Au} \mathrm{ions,} \mathrm{whereas} \mathrm{the} \mathrm{experiments} \mathrm{of}$ van Dillen were performed at a low temperature (85 K) using $4 \mathrm{MeV}$ Xe ions. Three factors can be invoked to account for the discrepancies observed: (i) the deposited energy, (ii) the temperature and (iii) the density of the material.

(i) The viscosity defines the resistance to flow (or deformation). In general, ion irradiation reduces the viscosity of the material. In particular, the larger the deposited energy, the larger the deformation effect. However, as for irradiation with $4 \mathrm{MeV} \mathrm{Au}$ ions, both nuclear and electronic stopping powers are larger than those relative to the irradiation with $4 \mathrm{MeV}$ Xe ions (table 1), hypothesis (i) can be excluded.

(ii) The temperature dependence $(90-575 \mathrm{~K})$ of $\eta_{\mathrm{RAD}}$ for thin thermally grown $\mathrm{SiO}_{2}$ film irradiated with $4 \mathrm{MeV}$ Xe ions has been studied by Brongersma et al [25]. They found that below about $300 \mathrm{~K}$, the irradiation-induced viscosity does not show a significant temperature dependence, 
Table 1. Characteristics of the irradiation conditions. $S_{\mathrm{e}}^{\mathrm{SiO}_{2}}$ is the electronic stopping power and $S_{n}^{\mathrm{SiO}_{2}}$ is the nuclear stopping power in silica as estimated from the code SRIM 2006 [15]. For $\mathrm{SiO}_{2}$ the density has been taken equal to $2.0 \mathrm{~g} \mathrm{~cm}^{-3}$.

\begin{tabular}{llll}
\hline Ion & Energy $(\mathrm{MeV})$ & $S_{\mathrm{e}}^{\mathrm{SiO}_{2}}\left(\mathrm{keV} \mathrm{nm}^{-1}\right)$ & $S_{n}^{\mathrm{SiO}_{2}}\left(\mathrm{keV} \mathrm{nm}^{-1}\right)$ \\
\hline $\mathrm{Au}^{2+}$ & 4 & 1.7 & 1.9 \\
$\mathrm{Xe}^{2+}$ & 4 & 0.79 & 1.56 \\
\hline
\end{tabular}

$\eta_{\mathrm{RAD}}=2.9 \times 10^{23} \mathrm{~Pa}$ ion $\mathrm{cm}^{-2}$, whereas above $300 \mathrm{~K}$, a decrease of $\eta_{\text {RAD }}$ is observed, e.g. at $575 \mathrm{~K}$ this value is reduced to $\eta_{\mathrm{RAD}}=1.6 \times 10^{23} \mathrm{~Pa}$ ion $\mathrm{cm}^{-2}$. As up to room temperature $\eta_{\mathrm{RAD}}$ remains constant, hypothesis (ii) can also be discarded.

(iii) Our estimation for $\eta_{\mathrm{RAD}}, 5.4 \times 10^{22} \mathrm{~Pa}$ ion $\mathrm{cm}^{-2}$, is between the value obtained by Brongersma for thermally grown silica film, $2.9 \times 10^{23} \mathrm{~Pa}$ ion $\mathrm{cm}^{-2}$, and that obtained by van Dillen for silica colloids, $0.9 \times$ $10^{22} \mathrm{~Pa}$ ion $\mathrm{cm}^{-2}$. In general, a thermally grown silica film is more dense than silica colloids, thus, under equivalent irradiation conditions, the value of $\eta_{\mathrm{RAD}}$ will be larger for the former specimen. On the other hand, the route followed during the chemical synthesis can lead to particles with different densities. Moreover, chemically synthesized NPs are not stable ad vitam aternam but evolve with time toward a different density state. Hence, the observed differences of $\eta_{\text {RAD }}$ could be attributed to differences in chemical preparation or to the evolution of the colloids with time.

3.1.2. Initial strain increment, $A_{0}$. The irradiation-induced anisotropic deformation is characterized by the deformation strain rate, i.e. the differential length change per unit fluence, $A_{0}=\mathrm{d} L /(L \mathrm{~d} \Phi)$, where $\Phi$ is the fluence and $L$ the dimension normal to the ion beam.

For thin metallic and silica glasses, irradiated at low temperature $(<100 \mathrm{~K})$ with high-energy ions (several $100 \mathrm{MeV}$ ), the value of $A_{0}$ was found to vary in the range of $10^{-14}-10^{-17} \mathrm{~cm}^{2}$ ion $^{-1}$ [17-19]. For silica colloids in the $100-1000 \mathrm{~nm}$ size range the deformation strain rate ranges between $A_{0}=6 \times 10^{-16} \mathrm{~cm}^{2}$ ion $^{-1}[20,11]$ and $A_{0}=$ $20 \times 10^{-17} \mathrm{~cm}^{2}$ ion $^{-1}$ [21]. However, we obtain an estimation for $A_{0}$ which is lower than the previous ones, i.e. $A_{0}=$ $0.90 \times 10^{-16} \mathrm{~cm}^{2}$ ion $^{-1}$. This difference may depend on the following factors: (i) the amount of energy deposited in the electronic sub-system $\left(S_{\mathrm{e}}\right)$, (ii) a temperature effect or (iii) the irradiation angle.

(i) Deformation strain rate, $A_{0}$, increases linearly with the electronic stopping power, $S_{\mathrm{e}}$. For $4 \mathrm{MeV} \mathrm{Au}$ ions and $4 \mathrm{MeV} \mathrm{Xe}$ ions within silica the value of $S_{\mathrm{e}}$ can be obtained using the code SRIM 2006 (table 1). As $S_{\mathrm{e}}^{4 \mathrm{MeV} \mathrm{Au}}>S_{\mathrm{e}}^{4 \mathrm{MeV} \mathrm{Xe}}$, the deposited energy effect can be excluded.

(ii) Previous studies have shown that, for $1000 \mathrm{~nm}$ silica colloids, $A_{0}$ decreases with temperature from about $6 \times$ $10^{-16} \mathrm{~cm}^{2}$ ion $^{-1}$ at $88 \mathrm{~K}$ to about $1 \times 10^{-16} \mathrm{~cm}^{2}$ ion $^{-1}$ at $370 \mathrm{~K}[20]$. This value approximately corresponds

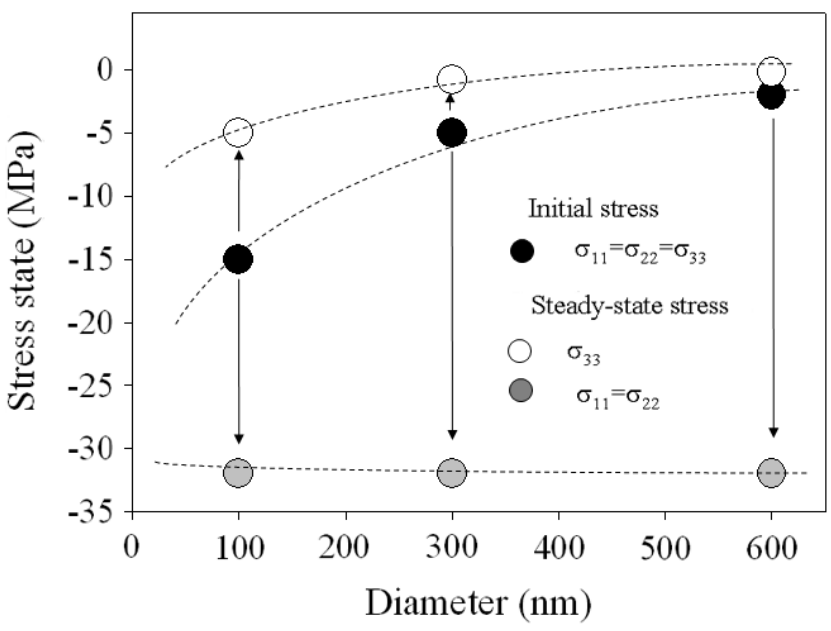

Figure 4. Initial hydrostatic stress, $\sigma_{11}(0)=\sigma_{22}(0)=\sigma_{33}(0)$ as a function of the NP size (full circles). In the same figure we added the steady-state stresses corresponding to the directions normal to the ion beam, $\sigma_{11}^{\text {steady-state }}=\sigma_{22}^{\text {steady-state }}$, (gray circles) and parallel to the ion beam, $\sigma_{33}^{\text {steady-state }}$ (open circles).

to our observation and validates the hypothesis that the reduction of the deformation strain rate is mainly due to a temperature effect, i.e. the higher the irradiation temperature, the lower the ion-hammering effect. This reduction of the strain rate with the temperature might be at the origin of the threshold fluence for deformation observed for $\mathrm{SiO}_{2}$ colloids.

(iii) Finally, Cheang-Wong et al [21] have shown that the deformation strain rate depends on the irradiation angle and exhibits a minimum of the order of $4 \times 10^{-17} \mathrm{~cm}^{-2}$ for irradiation at $45^{\circ}$ with $8 \mathrm{MeV} \mathrm{Si}$ ions. As this value is much larger than our observation, $A_{0} \sim 0.90 \times$ $10^{-16} \mathrm{~cm}^{2} /$ ion, this effect is unimportant in the present experiment.

\subsection{Evolution of the stress state inside the particle}

In this section, we estimate the initial stress state of the colloidal NPs and its evolution with the irradiation fluence.

The as-prepared $\mathrm{SiO}_{2}$ colloids are, in principle, shear stress-free. However, they are subject to a hydrostatic capillary pressure, $\sigma_{i i}(0)=\sigma_{11}(0)=\sigma_{22}(0)=\sigma_{33}(0)$, given by the Laplace equation:

$$
\sigma_{i i}(0)=-\frac{4 \gamma}{D_{0}}
$$

where $\gamma=0.4 \mathrm{~J} \mathrm{~m}^{-2}$ is the specific surface energy for colloidal silica [16], and $D_{0}$ is the initial diameter of the colloid. The value of $\sigma_{i i}(0)$ for the as-prepared NPs as a function of their initial size is reported in figure 4 (full circles). We obtain $\sigma_{i i}(0)=-15 \mathrm{MPa}$ for $100 \mathrm{~nm} \mathrm{NPs,} \sigma_{i i}(0)=$ $-5 \mathrm{MPa}$ for $300 \mathrm{~nm}$ NPs and $\sigma_{i i}(0)=-2.5 \mathrm{MPa}$ for $600 \mathrm{~nm}$ NPs. As the capillarity stress is function of the curvature radius, the smaller the NPs, the larger the initial hydrostatic pressure.

Under irradiation the sphere transforms into an oblate spheroid with the minor axis, $l(\Phi)$, along the ion beam and the 
major axis, $L(\Phi)$, normal to it. Fixing the irradiation parallel to the direction $a_{i}=a_{3}$, the stress along the ion beam is given by $\sigma_{33}(\Phi)$, whereas the stresses perpendicular to it are given by $\sigma_{11}(\Phi)=\sigma_{22}(\Phi)$. Assuming that all stress components are uniform and using the revolution symmetry of the particle, the evolution of the stress components $\sigma_{11}(\Phi)=\sigma_{22}(\Phi)$ and $\sigma_{33}(\Phi)$ reads [11]:

$$
\begin{gathered}
\sigma_{11}(\Phi)=\sigma_{22}(\Phi)=-2 \gamma \frac{1+(l(\Phi) / L(\Phi))^{2}}{L(\Phi)(l(\Phi) / L(\Phi))^{2}} \\
\sigma_{33}(\Phi)=-4 \gamma \frac{l(\Phi)}{L^{2}(\Phi)} .
\end{gathered}
$$

The value of the minor axis, $l(\Phi)$, has been estimated by combining TEM micrographs in tilted configuration and field emission scanning electron microscope (FE-SEM) images. The evolution of the principal stresses with fluence is represented in figures 5(a)-(c). Circles represent the evolution of the stresses perpendicular to the beam direction, $\sigma_{11}(\Phi)=$ $\sigma_{22}(\Phi)$, and triangles their evolution parallel, $\sigma_{33}(\Phi)$, to the beam direction.

Considering the steady-state stress along the beam, $\sigma_{33}^{\text {steady-state }}$, a saturation value of about $-5 \mathrm{MPa}$ is reached above a fluence of $0.5 \times 10^{16} \mathrm{~cm}^{-2}$ for $100 \mathrm{~nm} \mathrm{NPs}$ (full triangles, figure $5(\mathrm{a})$ ). This value is reduced to $-0.8 \mathrm{MPa}$ for fluences larger than $0.8 \times 10^{16} \mathrm{~cm}^{-2}$ for $300 \mathrm{~nm} \mathrm{NPs}$ (gray triangles, figure $5(\mathrm{~b})$ ) and to $-0.2 \mathrm{MPa}$ above $1.2 \times$ $10^{16} \mathrm{~cm}^{-2}$ for $600 \mathrm{~nm}$ NPs (open triangles, figure 5(c)). The evolution with the fluence for the stress normal to the beam, $\sigma_{11}(\Phi)=\sigma_{22}(\Phi)$, follows a sigmoidal-like curve indicating that the deformation process initially driven by the ion-track formation is progressively counterbalanced by the capillarity forces (open $(100 \mathrm{~nm})$, gray $(300 \mathrm{~nm})$ and full $(600 \mathrm{~nm})$ circles in figures 5(a)-(c)). The steady-state stress does not depend on the initial size of the NPs, i.e. $\sigma_{11}^{\text {steady-state }}=\sigma_{22}^{\text {steady-state }}=$ $-32 \mathrm{MPa}$. However, the saturation fluence does, i.e. the larger the NP size the larger the saturation fluence. Finally, the fact that the saturation fluence is the same for the internal stress, $\sigma_{i i}(\Phi)$, and the anisotropic deformation, $L(\Phi) / D_{0}$, indicates the tight correlation between these two effects.

\subsection{Evolution of the strain increment with fluence, $A(\Phi)$}

In general, the deformation strain rate is taken as a constant during the irradiation, i.e. the value of $A_{0}$ we estimated from equation (3). However, the validity of this approximation has only been checked for planar thin films. For small NPs, or, equivalently, for small values of the curvature radius, the macroscopic stress may modify the evolution of the strain increment with fluence. The relation between these two parameters, i.e. $A$ and $\sigma_{i j}$, can be derived from equation (2), ignoring the elastic contribution and using the definition of the strain rate in the direction of the major axis, $\mathrm{d} \varepsilon_{11} / \mathrm{d} \Phi=$ $\mathrm{d} L / L \mathrm{~d} \Phi$, and that of the deviatoric stress tensor $s_{i j}=\sigma_{i j}-$ $(1 / 3) \sigma_{k k} \delta_{i j}$. It is written:

$$
A(\Phi)=\frac{\mathrm{d} L}{L \mathrm{~d} \Phi}=A_{0}+\frac{\sigma_{11}(\Phi)-\sigma_{33}(\Phi)}{6 \eta_{\mathrm{RAD}}} .
$$
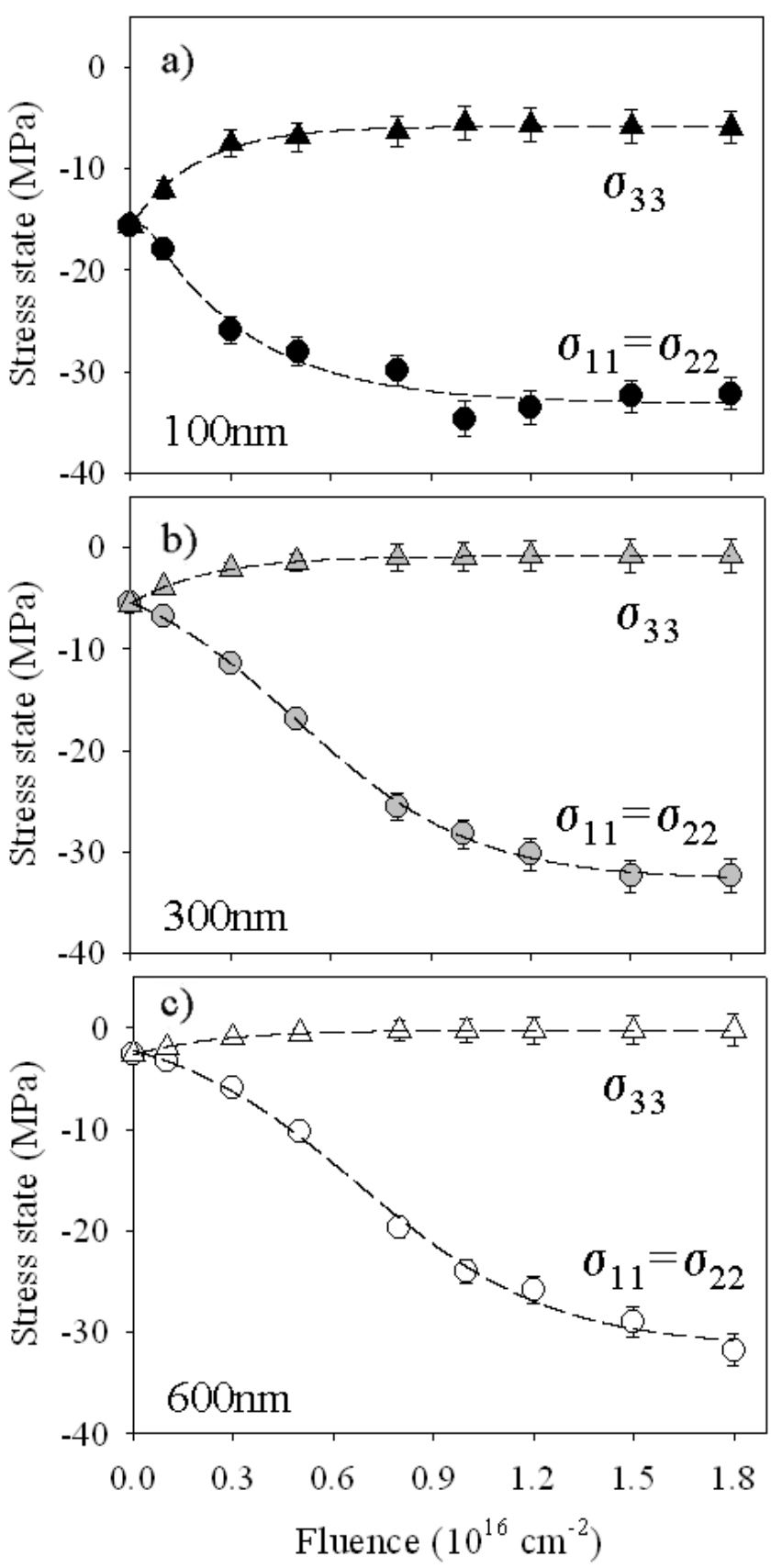

Figure 5. Evolution with fluence of the stress state normal to the ion beam, $\sigma_{11}(\Phi)=\sigma_{22}(\Phi)$ (circles) and parallel to the ion beam, $\sigma_{33}(\Phi)$ (triangles) for silica colloids: (a) $100 \mathrm{~nm} \mathrm{SiO}{ }_{2}$, (b) $300 \mathrm{~nm}$ $\mathrm{SiO}_{2}$ and (c) $600 \mathrm{~nm} \mathrm{SiO}_{2}$.

The first term on the right hand side represents the deformation strain rate in the absence of any stress, whereas the second term accounts for the contribution of the macroscopic stresses to the evolution of the strain increment with fluence. As these stresses are negative and $\left|\sigma_{11}\right|>$ $\left|\sigma_{33}\right|$, the ion-hammering decreases with fluence. When the two contributions exactly counterbalance each other the deformation strain rate becomes zero and the ion-deformation ends. Of course, when $\sigma_{i i} \rightarrow 0$, we obtain the classical expression for the deformation strain rate which is valid for thin films or large colloids. The evolution of $A(\Phi)$ can be obtained 


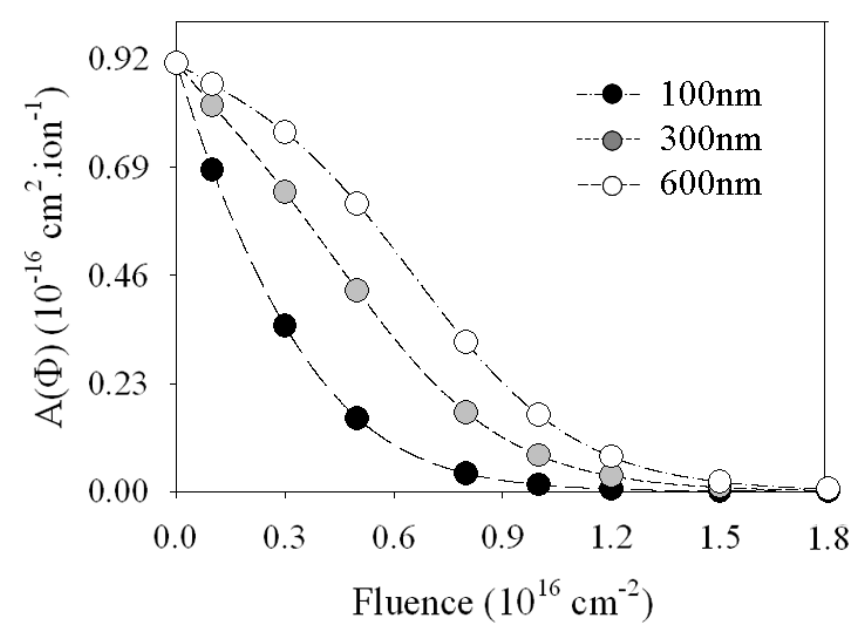

Figure 6. Evolution with fluence of the strain increment $A(\Phi)=\mathrm{d} L(\Phi) / L \mathrm{~d} \Phi$ for $\mathrm{SiO}_{2}$ colloids. The curves are the result of a calculation using equation (3) and the values of $A_{0}$ and $\eta_{\mathrm{RAD}}$ fitted on the normalized transverse diameters. $100 \mathrm{~nm}$ (full circles), $300 \mathrm{~nm}$ (gray circles) and $600 \mathrm{~nm}$ (open circles) colloidal NPs.

using $\mathrm{d} L / \mathrm{d} \Phi$ estimated from equation (3) and dividing it by the dimension normal to the ion beam, $L$. The result is shown in figure 6 for the three sizes of colloidal NPs. The value of $A(\Phi)$ rapidly decreases as a sigmoidal-like curve toward zero, i.e. for larger fluences the ion-hammering effect becomes negligible. The sigmoidal evolution of $A(\Phi)$ depends on the presence of the terms $\sigma_{i i}$ in equation (7). Thus, for sub-micrometer iondeformable colloidal NPs the approximation often made that $A(\Phi)$ is a constant is truly correct only for very low irradiation fluences. For example, a critical analysis of the van Dillen experiment shows that the reduction of $A(\Phi)$ is about $30 \%$ at a fluence of $1 \times 10^{15} \mathrm{~cm}^{-2}$.

\section{Conclusion}

In conclusion, the role of stresses due to the surface curvature in the ion-hammering phenomenon has been investigated using $\mathrm{SiO}_{2}$ colloids of different sizes as a model system. The samples were irradiated at room temperature with $4 \mathrm{MeV}$ $\mathrm{Au}$ ions for fluences in the range of $1.0 \times 10^{15}-1.8 \times$ $10^{16} \mathrm{~cm}^{-2}$. We show that the irradiation-induced deformation evolves toward a saturation value which depends on the initial size of the NPs. The larger the NP the larger the steady-state deformation as well as the irradiation fluence needed to reach this value. Experimental data were used to fit a phenomenological model for anisotropic deformation first developed by Klaumünzer [12] and further elaborated by van Dillen et al [11]. In particular, using the same set of fitted parameters, $A_{0}$ and $\eta_{\mathrm{RAD}}$, all the experimental curves are satisfactorily reproduced. We show that this model accounts for the evolution of the colloids up to a fluence of $1.8 \times 10^{16} \mathrm{~cm}^{-2}$, i.e. more than one order of magnitude larger than the fluences normally used to investigate the ionhammering effects. Thus, our observations represent the first demonstration that the capillarity stresses can compensate for the irradiation-induced stress, and thus counterbalance the ion-hammering effect. We also estimate the evolution with fluence of the macroscopic stresses inside the particles, i.e. $\sigma_{11}(\Phi)=\sigma_{22}(\Phi)$ and $\sigma_{33}(\Phi)$. Finally, we show that the approximation often made, that the deformation strain rate remains constant upon irradiation, is no longer valid for sub-micrometer colloidal particles and we derive an analytical expression that relates the strain rate, $A(\Phi)$, to the capillarity stresses, $\sigma_{i i}(\Phi)$. The extension of our observations to other systems will allow us to better characterize the ion-hammering when the non-hydrostatic capillary effects become important.

\section{Acknowledgment}

We wish to thank G Coddens (LSI-Ecole Polytechnique) for many fruitful discussions. Financial support by the French National Research Agency (ANR) for the Shaman project (ANR-09-BLAN-0334) is gratefully acknowledged.

\section{References}

[1] Klaumünzer S and Schumacher G 1983 Phys. Rev. Lett. 511987

[2] Cartz L, Karioris F G and Fournelle R A 1981 Radiat. Eff. 5457

[3] Klaumünzer S, Li C, Löffer S, Rammensee M, Schumacher G and Neitzert C H 1989 Radiat. Eff. Defects Solids 108131

[4] Klaumünzer S 1992 Mater. Sci. Forum 97-99 623

[5] van Dillen T 2006 PhD Thesis FOM Amsterdam, The Netherlands www.erbium.nl

[6] Trinkaus H and Ryazanov A I 1995 Phys. Rev. Lett. 745072

[7] Trinkaus H 1995 J. Nucl. Mater. 223196

[8] Trinkaus H 1998 Nucl. Instrum. Methods B 146204

[9] Eshelby J D 1957 Proc. R. Soc. A 241376

[10] van Dillen T, Polman A, Onck P R and van der Giessen E 2005 Phys. Rev. B 71024103

[11] van Dillen T, van der Giessen E, Onck P R and Polman A 2006 Phys. Rev. B 74132103

[12] Klaumünzer S 2004 Nucl. Instrum. Methods B 215345

[13] Stöber W, Fink A and Bohn E 1968 J. Colloid Interface Sci. 2662

[14] Khanh N N and Yoon K B 2009 J. Am. Chem. Soc. 13114228

[15] www.srim.org

[16] Scholze H and Lakin M J 1988 Glass: Nature, Structure and Properties 3rd edn (Berlin: Springer)

[17] Klaumünzer S 1989 Radiat. Eff. Defects Solids 11079

[18] Hou M-D, Klaumünzer S and Schumacher G 1990 Phys. Rev. B 411144

[19] Benyagoub A, Löffler S, Rammensee M, Klaumünzer S and Saemann-Ischenko G 1992 Nucl. Instrum. Methods B 65228

[20] van Dillen T, Polman A, van Kats C M and van Blaaderen A 2003 Appl. Phys. Lett. 834315

[21] Cheang-Wong J C, Morales U, Reséndiz E, Lopez-Suárez A and Rodríguez-Fernández L 2008 Nucl. Instrum. Methods B 2663162

[22] Volker C A and Polman A 1992 Mater. Res. Soc. Symp. Proc. 2353

[23] Snoek E, Polman A and Volker C A 1994 Appl. Phys. Lett. 652487

[24] Evangelakis G A, Rizos J P, Lagaris I E and Demetropoulos I N 1987 Comput. Phys. Commun. 46401

[25] Brongersma M L, Snoeks E and Polman A 1997 Appl. Phys. Lett. 711628 